\title{
A Case of Heart Failure with Preserved Ejection Fraction Secondary to Long-Term Clozapine Use
}

\author{
Mohammed Irshad Khan ${ }^{1 *}$, Ciaran McHale ${ }^{1}$, Aidan Stankard ${ }^{1}$ and Gerard Clarke ${ }^{1,2}$ \\ ${ }^{1}$ Portiuncula University Hospital, Ireland \\ ${ }^{2}$ Consultant Gastroenterologist, Ireland
}

*Corresponding author: Mohammed Irshad Khan, Portiuncula University

Hospital, Ballinasloe, Ireland.

Received Date: February 12, 2021

Published Date: March 01, 2021

\section{Summary}

We present the case of a 55-year-old female, on very low dose Clozapine treatment for Schizophrenia, who presented with shortness of breath and in atrial fibrillation and with no previous history of cardiac failure. Chest X Ray showed evidence of bilateral pulmonary oedema and bilateral pleural effusion, and a diagnosis of acute heart failure with preserved ejection fraction was made based on echocardiographic findings and raised brain naturetic peptide (BNP) levels. This was the patient's first presentation to hospital with heart failure related symptoms. Initial medical management included diuretics which did not improve her symptoms. With no other aetiology obvious her clozapine was stopped. Following this intervention her clinical status improved. Numerous case reports and systematic reviews have described cardiomyopathy associated with Clozapine use, but we describe below what we believe is the first documented case of Heart Failure with preserved Ejection Fraction (HFpEF) caused by Clozapine use.

\section{Background}

Schizophrenia is a serious brain disorder that has been estimated to have a lifetime prevalence of $0.48 \%$ [1]. Clozapine is the only medication licensed for the treatment of refractory Schizophrenia (defined as failure of at least 2 other anti-psychotic medications). It has been shown to have an impact on both the positive and negative symptoms of the disorder [2]. Serious recognised adverse effects to Clozapine treatment include agranulocytosis (prevalence of $1 \%$ of all treated patients) [3], toxic megacolon, and metabolic syndrome. The issue of Clozapine associated cardiomyopathy has been increasingly reported, with reports of incidences ranging from $0.02 \%$ in the UK [4] to $4.65 \%$ in a small Australian research report [5]. Wide regional and population variations are reported to exist. The cardiomyopathy described has invariably been a dilated cardiomyopathy $[3,8]$ with reduced ejection fraction [3]. The aetiology of the cardiomyopathy has not been definitively proven; myocarditis in the early period after Clozapine commencement has been hypothesised as a cause [8], as has a direct cardiotoxic Clozapine affect [10]. There is no consensus on the type of pre-treatment cardiac screening patients should have before commencing Clozapine, nor is there any consensus as to what ongoing cardiac monitoring patients should have during their Clozapine treatment. At the very minimum, a baseline ECG and exploration of previous cardiac history are generally advised. Concerns have been raised that broadening pre-treatment cardiac screening may be costly and could lead to unnecessary barriers to Clozapine prescription $[7,9]$.

\section{Case Presentation}

A 56-year-old female smoker presented to the emergency department with a background history of schizoaffective psychosis, hypertension, anti-phospholipid syndrome, depression, alcohol misuse, COPD, and right thyroid lobectomy. Of note, she was taking warfarin for 15 years following a DVT diagnosed 15 years previously as well as clozapine 100mg nocte since 2009 for treatment resistant schizophrenia. She had a 45-pack year smoking history. On presentation the patient described a gradually 
worsening shortness of breath for one week. This was associated with a productive cough. She was recently treated by her GP for a lower respiratory tract infection with antibiotics for two weeks. On initial examination she was found to be in new fast atrial fibrillation (140bpm), hypotensive and requiring 5L of oxygen to maintain saturations above $92 \%$. Auscultation of the chest revealed bi-basal crackles and mild expiratory wheeze was heard. Heart sounds I+ II were heard with no added sounds. GCS was 15/15. Neurological exam was normal.

\section{Investigations}

Investigations revealed a total white cell count of $18.2 \times 109 / \mathrm{L}$ (normal 3.9-11.1x100/L) and neutrophilia of 15.5x109/L (normal 1.7-7.5x109/L). CRP was slightly elevated at $11.8 \mathrm{mg} / \mathrm{L}$ (normal $0-5 \mathrm{mg} / \mathrm{L}$ ). A U\&E showed an AKI with a creatinine of 134umol/L. Arterial blood gas were as follows; pH 7.46, pCO2 5.39, p02 7.28, HCO3 28.7, SpO2 89.9\%. Initial chest x-ray demonstrated cardiomegaly, a finding noted on a film taken one-year prior moderate bilateral pleural effusions and bilateral lower lobe pneumonic airspace disease in keeping with consolidation. Two days following initial presentation a high resolution CT thorax demonstrated bilateral pleural effusions. Bilateral lower lobe bronchial wall thickening, and mucous plugging were also noted. In addition, a pro-BNP taken on admission returned at $>5822 \mathrm{pg} / \mathrm{mL}$. An ECHO showed overall normal left ventricular (LV) and systolic function (Ejection fraction estimated at 59\%). The right ventricle (RV) was mildly dilated, with preserved function. Mild to moderate aortic regurgitation (AR) and mild tricuspid regurgitation were also noted. Of note, an ECHO recorded 14 years previous noted normal LVF and mild AR

\section{Differential Diagnosis}

The differential diagnoses considered during this case, were respiratory sepsis, acute decompensated heart failure (HFpEF) and COPD.

\section{Treatment}

The patient was admitted to ICU where she received intravenous antibiotics and diuresis oxygen therapy and a phenylephrine infusion to maintain blood pressure. However, the patient did not respond to initial medical management. With no other obvious cause for her heart failure evident, clozapine was stopped. A significant improvement in status followed this intervention. Initially, digoxin was used to control her fast atrial fibrillation. She then received DC cardioversion 10 days following admission, which restored sinus rhythm. Monitoring of daily weights and clinical symptoms was used to guide diuresis. Once stabilized medications such as eplerenone, carvedilol, ramipril and bumetanide were commenced. Amiodarone was also started prior to discharge.

\section{Outcome and Follow Up}

A diagnosis of types 1 respiratory failure secondary to acute decompensated heart failure was made. This previously undiagnosed heart failure is believed to be a result of long term clozapine therapy. The patient responded well to treatment and was discharged ten days following first presentation. Persistent hypoxemia fulfilled criteria for long-term oxygen therapy. She attended cardiology outpatients one month later as well as follow up appointments with local heart failure services where an improvement was noted.

\section{Discussion}

To our knowledge this is the first written case of heart failure with preserved ejection fraction due to clozapine usage. Previous case reports have described the development of heart failure with reduced ejection fraction. In our literature review we found three systematic reviews $[2,7,11]$ focusing on cardiomyopathies associated with Clozapine usage. None of these have noted HFpEF as a clinical finding in clozapine associated cardiomyopathies. Our investigations revealed evidence of right sided heart failure with preserved ejection fraction. With no other obvious etiological factors evident we believe this patient's presentation was caused by heart failure secondary to clozapine therapy. The existing literature and previous case reports on Clozapine-associated cardiac side effects have focused on heart failure and cardiomyopathies earlier in the course of clozapine treatment. Previous studies also report adverse effects associated with larger doses of Clozapine. Our patient developed heart failure after prolonged treatment of very low dose Clozapine. Further research is needed into the long term cardiac side effect profile of Clozapine and whether these side effects are dose dependent or independent.

For a full diagnostic evaluation, it would be ideal to perform a Cardiac MRI to fully evaluate the myocardial structure. However, our patient did not attend for cardiac MRI. Although evidence of heart failure was evident on plain film XR one year prior to this emergency presentation, she had not, on any previous occasions, presented with any other clinical manifestations of heart failure/ cardiomyopathy. This was nine years after commencing Clozapine. This is significantly longer than the reported median time of diagnosis of cardiomyopathy in a recent systematic review of Clozapine related cardiomyopathy [2]. Furthermore, our patient had been taking $100 \mathrm{mg}$ of Clozapine once a day, dosage which has been termed "very low dose" in the literature [11]. In the literature, although cardiomyopathy has been described as a dose-independent consequence of clozapine treatment [6], those diagnosed with clozapine associated cardiomyopathy have been using a much higher median dosage [3] In the described case, our patient has not been re-challenged with Clozapine therapy. Successful reintroduction of Clozapine after dilated cardiomyopathy has been described in the literature [10], however there has also been a reported case of the recurrence of cardiomyopathy following reintroduction. 


\section{Acknowledgement}

None.

\section{Conflict of Interest}

No conflict of interest.

\section{References}

1. Rotella P, Collins J, Windisch R (2018) An evaluation of variation in published estimates of schizophrenia prevalence from 1990-2013: a systematic literature review. BMC Psychiatry 193(15).

2. Alawami M, Wasywich C, Cicovic A, Kenedi C (2014) A systematic review of clozapine induced cardiomyopathy. Int J Cardiol 176(2): 315-320.

3. Longhi S, Heres S (2017) Clozapine-induced, dilated cardiomyopathy: a case report. BMC Res Notes 10(1): 338.

4. Curto M, Girardi N, Lionetto L, Ciavarella G, Ferracuti S, et al. (2016) Systematic Review of Clozapine Cardiotoxicity. Curr Psychiatry Rep 18(7): 68.

5. Youssef D, Narayanan P, Gill N (2015) Incidence and risk factors for clozapine-induced myocarditis and cardiomyopathy at a regional mental health service in Australia. Australas Psychiatry 24(2): 176-180.
6. De Berardis D, Rapini G, Olivieri L, Di Nicola D, Tomasetti, C, et al. (2018) Safety of antipsychotics for the treatment of schizophrenia: a focus on the adverse effects of clozapine. Ther Adv Drug Saf 9(5): 237-256.

7. Knoph K, Morgan R, Palmer B, Schak K, Owen A, et al. (2018) Clozapine-induced cardiomyopathy and myocarditis monitoring: A systematic review. Schizoph Res 199: 17-30.

8. Kilian J, Kerr K, Lawrence C, Celermajer D (1999) Myocarditis and cardiomyopathy associated with clozapine. The Lancet 354(9193): 18411845.

9. Stuart Murch, Nga Tran, Danny Liew, Melissa Petrakis, David Prior, et al. (2013) Echocardiographic monitoring for clozapine cardiac toxicity: lessons from real-world experience. Australas Psychiatry 21(3): 258-261.

10. Nederlof M, Benschop T, de Vries Feyens C, Heerdink, E (2017) Clozapine re-exposure after dilated cardiomyopathy. BMJ Case Rep 2017: bcr2017219652.

11. Merrill D, Dec G, Goff D (2005) Adverse Cardiac Effects Associated With Clozapine. Journal of Clinical Psychopharmacology 25(1): 32-41. 